Check for updates

Cite this: RSC Adv., 2018, 8, 42292

\title{
Polymer ionic liquid network: a highly effective reusable catalyst for one-pot synthesis of heterocyclic compounds $\uparrow$
}

\begin{abstract}
Xinjuan Li, (D) * Shangyue Wang, Kai Wang, Xianbin Jia and Zhiguo Hu (D) *
Significant efforts have been devoted to developing immobilized chiral catalysts with high activity, selectivity, and stability. In this present study, a new heterogeneous proline catalyst system was prepared based on strong noncovalent interactions between polymer ionic liquid (PIL) and L-proline. First, pyridine PILs, which can complex with L-proline monomers through noncovalent interactions, were synthesized using reversible addition-fragmentation chain transfer (RAFT) polymerization. The polymer network-supported chiral catalysts were obtained following further free radical polymerization. Different structures were formed in response to different ratios of PIL and chiral monomer, as well as different PIL anions, in the reactions. The new formed layer structures and synergic effects of PIL resulted in heterogeneous catalysts with high catalytic activity and enantioselectivity, thus endowing them with better catalytic performance for the one-pot synthesis of heterocyclic compounds compared to homogeneous catalytic systems. These catalytic systems were able to be reused and recycled five times with no discernible loss in catalytic activity and enantioselectivity. L-Proline was efficiently loaded onto the polymer network simply based on supramolecular interactions, providing a novel method of synthesizing high performance supported catalysts for organic reactions.
\end{abstract}

Received 21st October 2018

Accepted 9th December 2018

DOI: 10.1039/c8ra08712a

rsc.li/rsc-advances disadvantages and has been the focus of considerable work in recent years. ${ }^{\mathbf{1 0 - 1 6 , 3 1 - 3 4}}$ Supporting solids commonly used include polymer, ${ }^{17,18}$ silica, ${ }^{19,21}$ ionic liquid, ${ }^{23-29}$ merrifield resin, ${ }^{22}$ and magnetite. ${ }^{20}$ It remains a challenge to prepare highly efficient and recyclable chiral catalyst. Ionic liquids have been used as carriers in catalyst loading, ${ }^{24,30}$ where they can act as regulators to improve the efficiency and reusability of chiral catalyst in direct asymmetric aldol reactions. ${ }^{25,26}$ However, the separation of IL-tagged catalyst is an undesirable process. Polymer ionic liquid (PIL) is a polymer with an ionic liquid repeat unit that possesses the unique characteristics of polymers and ionic liquids containing anions and cations. ${ }^{2}$ Compared with small molecule ionic liquids, it is easier to use PIL to prepare effective load catalytic systems due to its variable long-chain structure. Our team has prepared nanoparticle-supported L-prolinamide catalyst based on PIL using in situ ionic complexation. ${ }^{2}$ The PILmodified solid catalytic systems have advantages in terms of separating and accelerating asymmetric reactions.

In this present study, we illustrate a novel concept for the preparation of the heterogeneous proline catalyst system which was prepared based on the strong noncovalent interactions between PIL and L-proline. Pyridine PILs with well-controlled architectures formed via reversible addition-fragmentation chain transfer (RAFT) polymerization can create strong noncovalent bonds with L-proline monomer due to the non-covalent interactions between L-proline and PIL. ${ }^{35}$ Furthermore, the catalytic networks were formed through one step of free radical polymerization (Fig. 1). The obtained catalysts were 


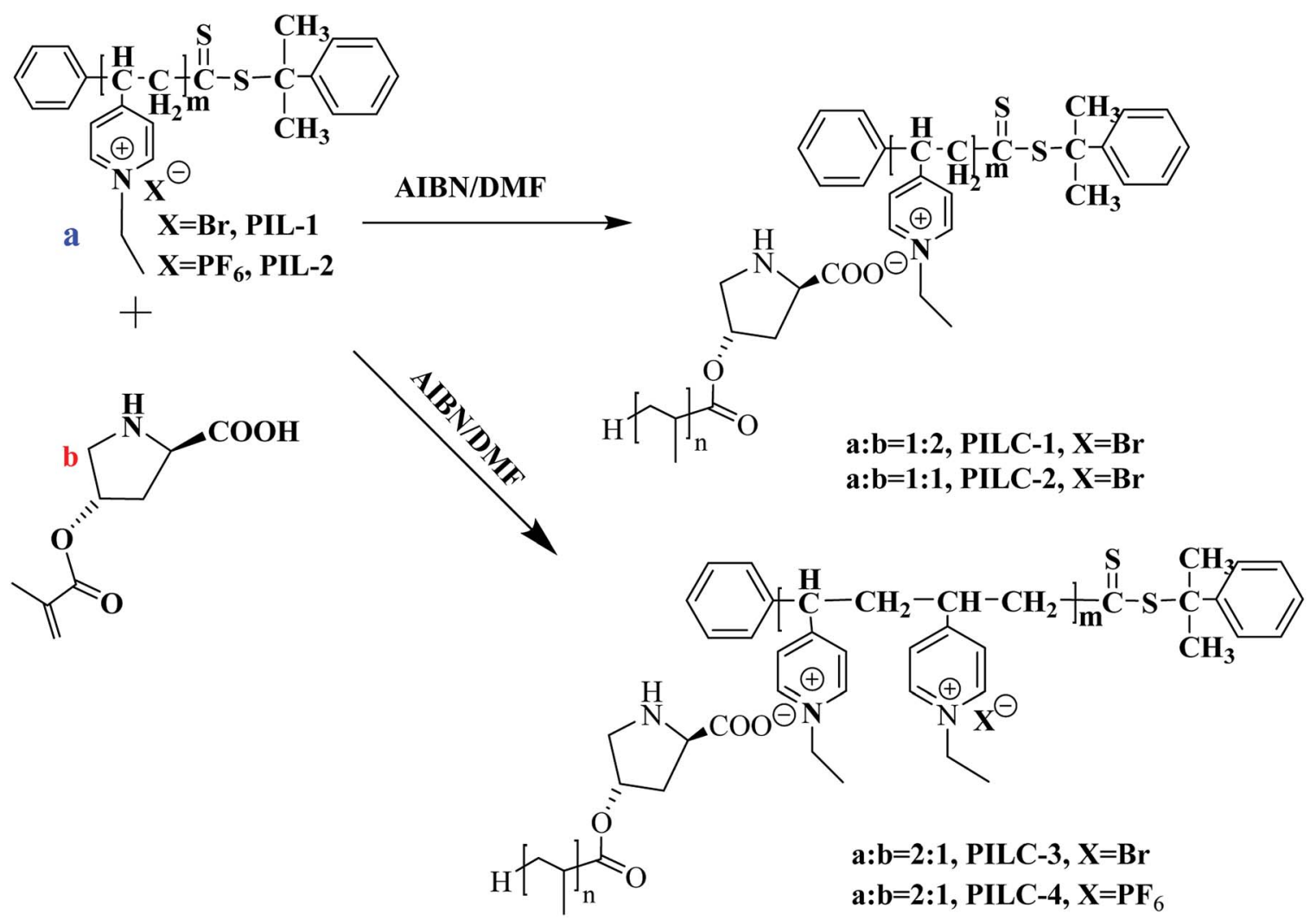

Fig. 1 Synthesis of heterogeneous catalyst-supported L-proline based on supramolecular interactions and free radical polymerization.

characterized using Fourier transform infrared spectra (FT-IR), scanning electron microscopy (SEM), X-ray diffraction (XRD), Xray photoelectron spectroscopy (XPS), and elemental analysis. The influences of different proportions of PIL and proline monomer, as well as PIL anion structure, on catalytic activity and asymmetric selectivity were investigated through aldol reactions and MCRs. It was found the PIL complex provides a new layer structures and synergic effects that increase the catalytic performance of the heterogeneous s-proline catalyst. In this present study, L-proline was efficiently loaded onto the polymer network through noncovalent interactions and free radical polymerization, thus providing a new approach to synthesizing high performance supported catalysts for use in organic reactions.

\section{Experimental section}

Materials and methods

The chemicals 2-chlorobenzaldehyde, 4-nitrobenzaldehyde, and 4-acetamido benzaldehyde were purchased from TCI. Azobisisobutyronitrile (AIBN) was recrystallized in ethanol. $\mathrm{N}$ Dimethylformamide (DMF) was purified by distillation after drying. Aromatic aldehyde and 4-vinyl pyridine (4-VP) were purified by distillation. L-Proline monomer and cumyl dithiobenzoate $(\mathrm{CDB})$ were prepared according to a previous publication. ${ }^{2}$

${ }^{1} \mathrm{H}$ NMR spectra were obtained using an NMR spectrometer (Bruker $400 \mathrm{MHz}$ ). A Thermo FLASH 1112 elemental analyzer was used for elemental analysis. Infrared spectroscopy and HPLC were performed on a Fourier transform-infrared spectrometer (Nicolet NEXUS) and an Agilent TM 1100, respectively. The molecular weight distribution (PDI $=M_{\mathrm{w}} / M_{\mathrm{n}}$ ) and molecular weight of the synthesized polymer were measured by gel permeation chromatography using a Waters 1515 apparatus, DMF as the eluent at a flow rate of $1.0 \mathrm{~mL} \mathrm{~min}^{-1}$, and polystyrene samples as standards. Field emission scanning electron microscopy (NovaNano SEM450) were used to characterize the morphology of the catalyst. XPS spectra were obtained on a VG ESCALAB MK II spectrograph.

\section{Preparation of heterogeneous catalyst-supported L-proline}

Preparation of PILs. CDB $(20.7 \mathrm{mg}, 0.076 \mathrm{mmol}), 4-\mathrm{VP}$ (0.40 g, $3.85 \mathrm{mmol})$, AIBN (3.1 mg, $0.019 \mathrm{mmol})$, and DMF (5 $\mathrm{mL}$ ) were combined in a $10 \mathrm{~mL}$ round-bottom flask. After stirring for $10 \mathrm{~min}$, a clear solution was obtained. Oxygen was completely removed from the system with nitrogen and then the flask was sealed and incubated at $65{ }^{\circ} \mathrm{C}$ with stirring. After allowing the reaction to proceed for $48 \mathrm{~h}$, the product was diluted in ether, centrifuged, washed with ether to remove 4-VP monomer, and dried at $40{ }^{\circ} \mathrm{C}$ to obtain P4VP with the yield of $66 \%$.

After dissolving P4VP ( $0.1 \mathrm{~g}, 0.95 \mathrm{mmol})$ in $3 \mathrm{~mL}$ chloroform, $9.5 \mathrm{mmol}$ bromoethane was added to the flask and the mixture was heated to $60{ }^{\circ} \mathrm{C}$ for $48 \mathrm{~h}$. After the mixture had cooled, the solid was thoroughly washed with chloroform to remove unreacted P4VP and the obtained solid was dried under 

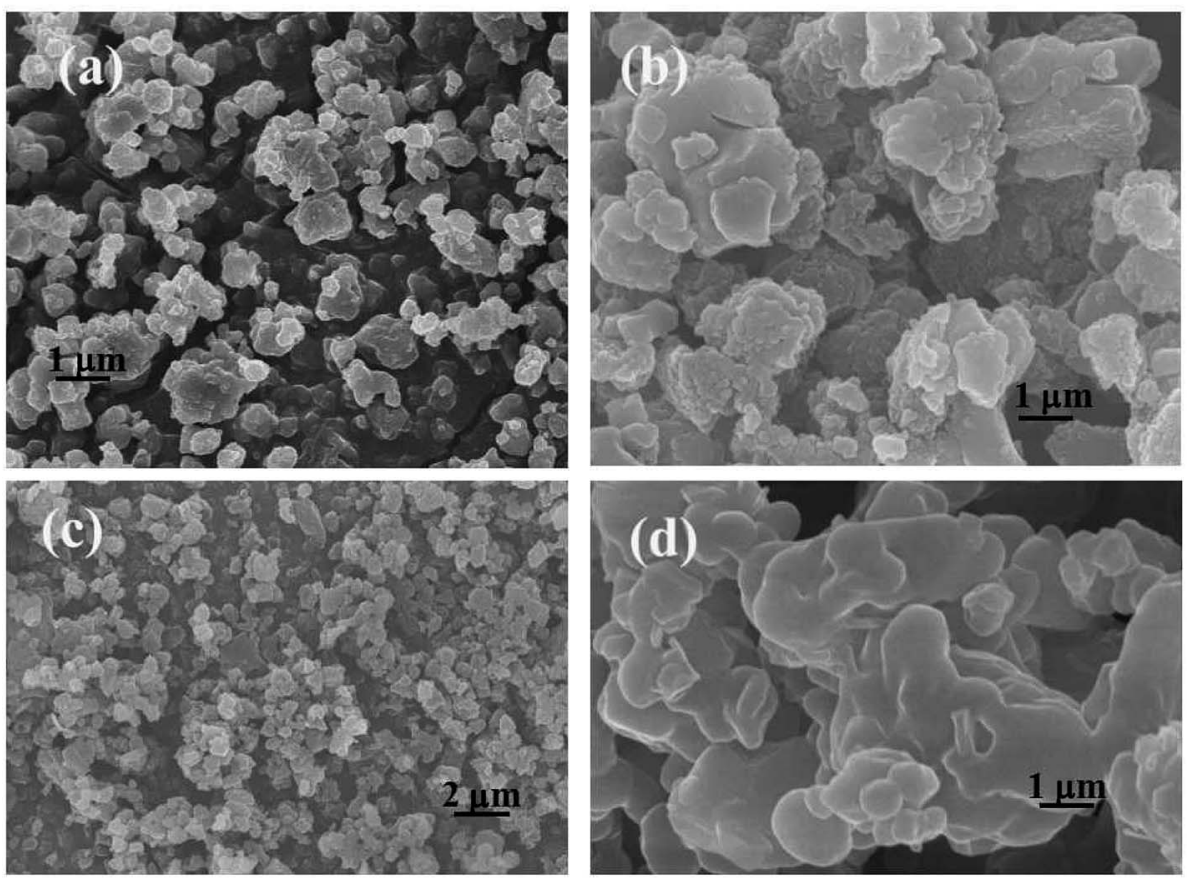

Fig. 2 SEM images of catalyst (a) PILC-1, (b) PILC-2, (c) PILC-3, and (d) PILC-4.

pressure to create PIL-1. The average molecular weight was 5301 and the PDI $=1.29$ (gel permeation chromatography analysis).

PIL-2 with hydrophobic anion $\left(\mathrm{PF}_{6}{ }^{-}\right)$was also prepared using a standard procedure. PIL-1 (29.30 g, $0.067 \mathrm{mmol})$ and $\mathrm{KPF}_{6}(12.33 \mathrm{~g}, 0.067 \mathrm{mmol})$ were dissolved in $100 \mathrm{~mL}$ distilled water, which formed a clear solution, and then stirred at $25{ }^{\circ} \mathrm{C}$ for $5 \mathrm{~h}$ until an insoluble oily substance was formed, which was collected by centrifugation. The crude product was washed first with water and then ether and dried at $60{ }^{\circ} \mathrm{C}$ under a vacuum.

\section{Synthesis of poly(ionic liquid) complex networks}

Poly(ionic liquid) complexes (PILCs) were prepared as follows: $0.02 \mathrm{~g}$ PIL-1 (2 mmol) and $0.40 \mathrm{~g}$ L-proline monomer $(2 \mathrm{mmol})$ were dissolved in $10 \mathrm{~mL}$ of DMF and then $2.2 \mathrm{mg}$ AIBN $(0.0134$ $\mathrm{mmol}$ ) were added. In order to remove oxygen from the system, degassing was carried out using five freeze and thaw cycles and then the sealed bottle was put into an oil bath at $75^{\circ} \mathrm{C}$ for $48 \mathrm{~h}$. The product was collected by centrifugation and washed with DMF and ethanol 5 times to remove unreacted monomer and the free polymer. The resulting light blue product was dried at $40{ }^{\circ} \mathrm{C}$ for $48 \mathrm{~h}$ to obtain PILC- 1 with the yield of $80 \%$.

PILC-2, which contained a molar ratio of PIL-1 to proline monomer of $2: 1$, and PILC-3, which contained a molar ratio of PIL-1 and proline monomer of $1: 2$, were synthesized in a manner similar to PILC-1, except using different molar ratios of PIL and proline monomer.

Synthesis of PILC-4 was similar to PILC-3 except PIL-2 $\left(\mathrm{PF}_{6}{ }^{-}\right)$ was used.

\section{PILC application in asymmetric reactions}

PILCs were applied in asymmetric aldol reactions of ketone with 2-nitrobenzaldehyde.

Table 1 Chemical composition of different PIL networks determined by elemental analysis

\begin{tabular}{|c|c|c|c|c|c|}
\hline Sample & Yield (\%) & $\mathrm{N}$ content (wt\%) & $\mathrm{S}$ content (wt\%) & L-Proline content ${ }^{a}\left(\mathrm{mmol} \mathrm{g}^{-1}\right)$ & Chiral polymer mol ratio $: \mathrm{PIL}^{b}$ \\
\hline PIL-1 & & 5.24 & 0.71 & 0 & \\
\hline PILC-1 & 87 & 6.23 & 0.47 & 1.99 & $1.25: 1$ \\
\hline PILC-2 & 86 & 5.89 & 0.37 & 2.23 & $1: 1.16$ \\
\hline PILC-3 & 80 & 6.09 & 0.33 & 2.61 & $1: 1.51$ \\
\hline PILC-4 & 92 & 5.65 & 0.43 & 1.70 & $1: 1.26$ \\
\hline PILC- $3^{c}$ & & 6.02 & 0.32 & 2.60 & \\
\hline
\end{tabular}

${ }^{a}$ Calculated by the elemental analysis results, and L-proline content was calculated according to the following formula: $\left(\frac{x}{M_{\mathrm{N}}}-\frac{m y}{M_{\mathrm{S}}}\right) / 100, x$ represents $\mathrm{N}$ content and $y$ represents S content, $m=\frac{x_{\mathrm{PIL}-1}}{M_{\mathrm{N}}} / \frac{y_{\mathrm{PIL}-1}}{M_{\mathrm{S}}} \cdot{ }^{b}$ Calculated according to the following formula: $\frac{m y}{M_{\mathrm{S}}}:\left(\frac{x}{M_{\mathrm{N}}}-\frac{m y}{M_{\mathrm{S}}}\right) \cdot{ }^{c} \mathrm{PILC}-$ 3 was tested after 5 cycles determined by elemental analysis. 


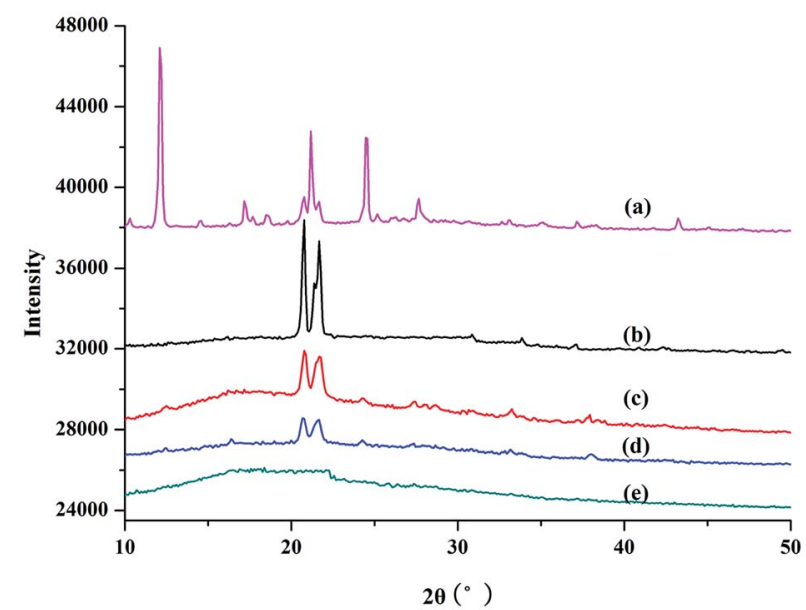

Fig. 3 XRD patterns of (a) L-proline, (b) PILC-1, (c) PILC-2, (d) PILC-3, and (e) PILC-4.

Acting as catalysts, PILCs (10 mol\%, $0.01 \mathrm{mmol}$ proline content) were added to a mixture of acetone $(104 \mu \mathrm{L}, 1.0 \mathrm{mmol})$ and 4-nitrobenzaldehyde $(0.25 \mathrm{mmol}, 38 \mathrm{mg})$ in $1 \mathrm{~mL}$ of the corresponding solvent. The mixtures were stirred at different temperatures and monitored by thin-layer chromatography (TLC) until the reactions were complete. The reaction mixtures were isolated by centrifugation and the PILCs were washed with methanol. The solids were dried under a vacuum for the next cycle. The aqueous layers were extracted with EtOAc and then dehydrated with $\mathrm{MgSO}_{4}$. After evaporation of the solvent, the crude products were separated and purified by column chromatogram (petroleum ether/EtOAc $=4: 1, \mathrm{v} / \mathrm{v}$ ) to yield the desired products.

\section{PILCs applied in MCRs}

Acting as catalysts, PILCs ( $20 \mathrm{~mol} \%, 0.2 \mathrm{mmol}$ proline content) were added to a mixture of 2-hydroxy-1,4-naphthoquinone (1

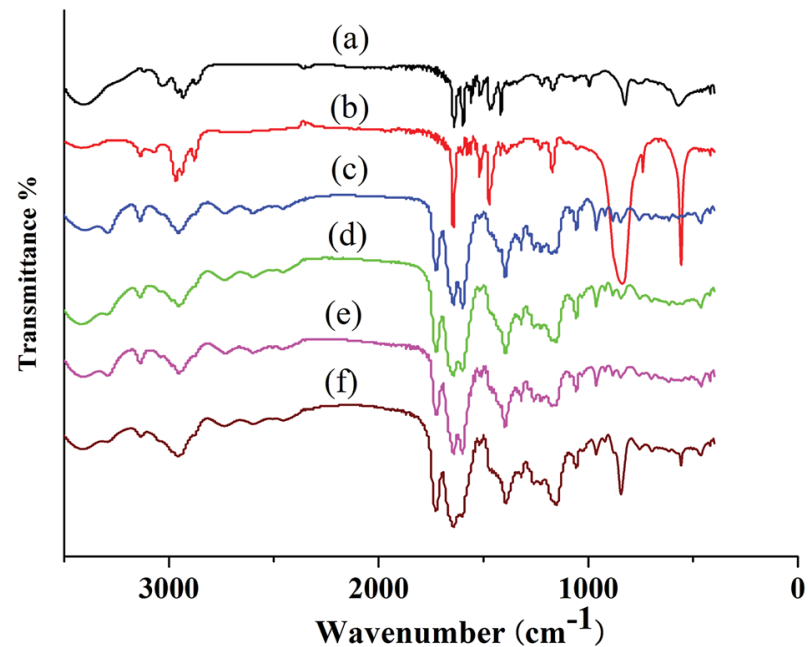

Fig. 4 Fourier transform-infrared spectra of (a) PIL-1, (b) PIL-2, (c) PILC-1, (d) PILC-2, (e) PILC-3, and (f) PILC-4.

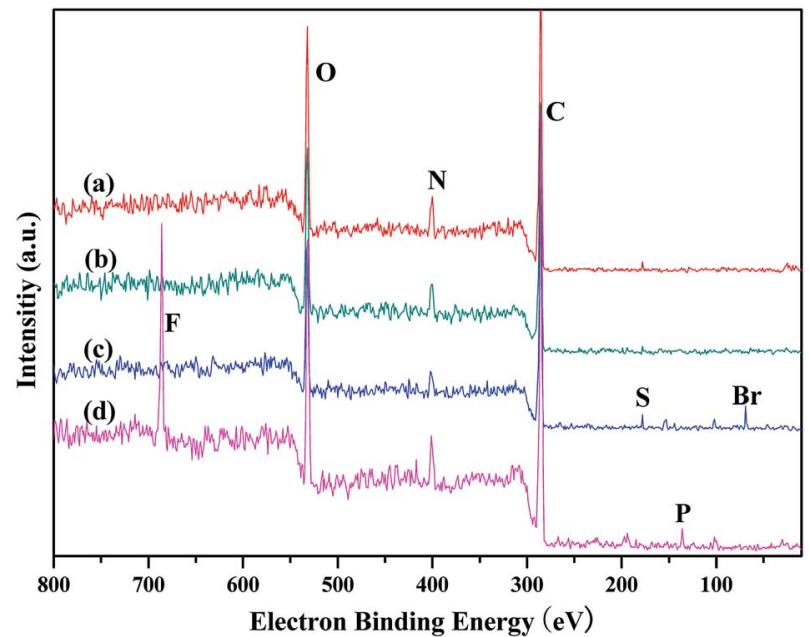

Fig. 5 XPS of (a) PILC-1, (b) PILC-2, (c) PILC-3, and (d) PILC-4.

mmol) and aldehyde ( $1 \mathrm{mmol})$ in the corresponding $1 \mathrm{~mL}$ solvent. The mixture was heated to $80{ }^{\circ} \mathrm{C}$ with stirring for 30 minutes. After the addition of 1 mmol 3-amino-5methylpyrazole, the reaction continued with stirring until complete as monitored by TLC. The resulting solid was filtered and purified using ethanol. The PILC catalysts were then dissolved in tetrahydrofuran, filtered, and washed with ethanol for the next cycle. The aqueous layer was then evaporated to yield the desired product.

\section{Results and discussion}

\section{Preparation of the heterogeneous catalyst-supported L-proline}

In our previous research, PILs were successfully used for highly sensitive detection of amine acid in solution. The biosensitivity of PIL was dependent on the carboxyl and secondary amine groups of L-proline, which can form ionic bonds and hydrogen

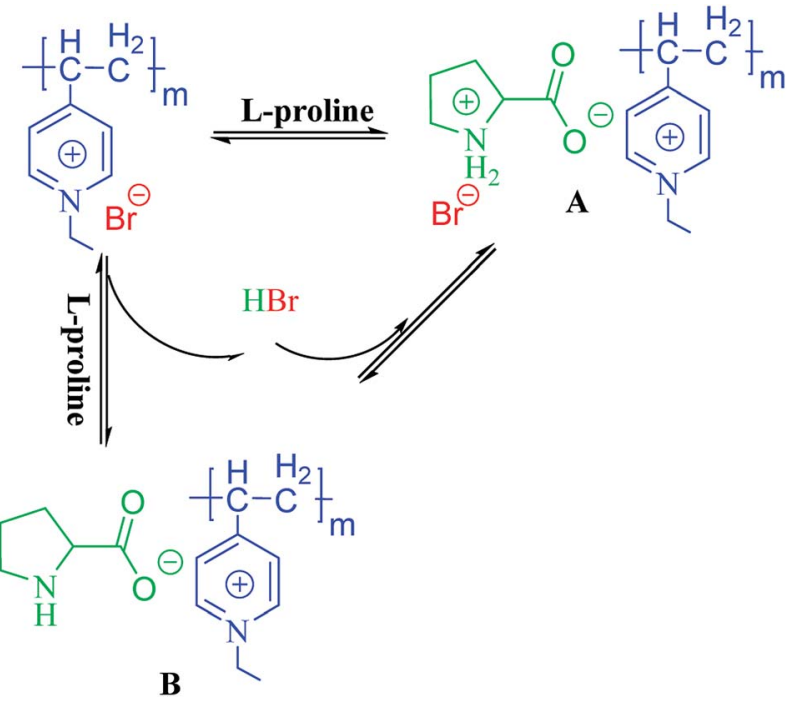

Scheme 1 Species for the direct interaction of (L)-proline with PIL-1. 
Table 2 Aldol reaction between acetone and 4-nitrobenzaldehyde catalyzed by different catalyst systems for $24 \mathrm{~h}$ in DMF

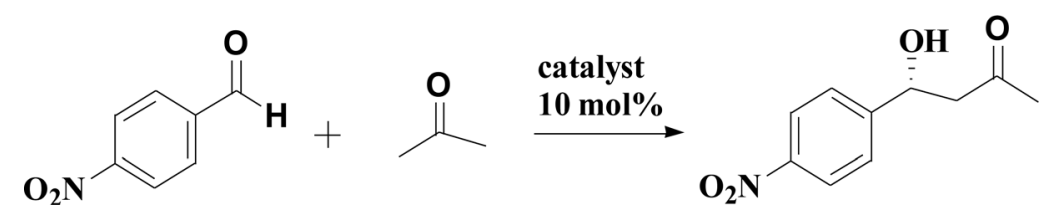

\begin{tabular}{llll}
\hline Catalysts & Temperature $\left({ }^{\circ} \mathrm{C}\right)$ & $\%$ conversion $^{a}$ & \% ee ${ }^{b}$ \\
\hline L-Proline & 25 & 55 & 76 \\
Proline pyridinium salt & 25 & 35 & 71 \\
PIL-1 + L-proline (mol ratio 1:1) & 25 & 55 & 78 \\
PILC-1 & 25 & 80 & 60 \\
PILC-2 & 25 & 80 & 77 \\
PILC-3 & 25 & 82 & 70 \\
PILC-4 & 25 & 37
\end{tabular}

${ }^{a}$ Determined by ${ }^{1} \mathrm{H}$ NMR spectroscopic analysis of the product. ${ }^{b}$ Determined by HPLC using a chiral column.

bonds with PIL. ${ }^{34}$ The polymer network supported L-proline was obtained by mixing PIL with L-proline monomer in DMF solution and further free radical polymerization. In addition, we also reported that PIL had synergic effects and improved the performance of the catalytic system. ${ }^{3}$ Therefore, we synthesized a series of complex networks by modifying the reaction ratios of PIL and L-proline monomer and PIL anion to study the influence of ionic liquid polymer on the performance of the catalysts.

\section{Sample characterization}

Fig. 2 presents the microstructure and morphological evolution of the heterogeneous catalyst. SEM images of PILC-1, which appeared as sheets with a polygonal morphology, a length of approximately 800-900 nm, are shown in Fig. 2a. Fig. 2b presents the microscopic structure and morphological evolution of PILC-2, where also formed a heterogeneous layer structure. When more PIL added than proline monomer was added (as in Fig. 2c, where the molar ratio was 1.25 for PIL and proline monomer, as shown in Table 1) as for PILC-3, the PILC matrix formed a heterogeneous layer structure. Overall, the individual proline polymers were homogeneously dispersed throughout the PIL network and the proline polymer was physically absorbed onto the surface of the PIL, assembling into a new structure during the simple process of radical polymerization. PILC-4 with $\mathrm{PF}_{6}{ }^{-}$anion formed a homogeneous amorphous structure (Fig. 2d), where the proline polymer had fully permeated into the PIL matrix and formed no regular structure.

XRD was used to study the crystal and structural changes during polymerization. Fig. 3 shows the XRD results for PILC and L-proline monomer. The main intense diffraction peaks for proline monomer appeared at $12.09^{\circ}(003)$ and $21.17^{\circ}(006)$, which is in accordance with a previous report concerning these amino acids (Fig. 3a). ${ }^{32,33}$ In Fig. 3b-d for PILC-1 and PILC-2, the diffraction peaks of L-proline had almost completely disappeared and two new peaks appeared at 20.77 and $21.67^{\circ}$ that corresponded to average interlayer distances of $c a .0 .42 \mathrm{~nm}^{32}$ The XRD of PILC-4 revealed the disappearance of the diffraction peak corresponding to proline and there were no other peaks in the complex (Fig. 3e), which may be due to the formation of an amorphous structure and the crystal structure was completely inhibited.

FT-IR spectroscopy was used to evaluate the structure of PIL complex (Fig. 4). The FT-IR spectrum of PILC (1-4) included characteristic vibration bands corresponding to $\mathrm{C}=\mathrm{O},-\mathrm{OH}$, and $-\mathrm{NH}$ at 1719, 3310, and $1050 \mathrm{~cm}^{-1}$ respectively (Fig. 4c-f). The characteristic band at $1625 \mathrm{~cm}^{-1}$ relating to the $\mathrm{C}=\mathrm{O}$ of the carboxyl group of the proline unit and the band at $1600 \mathrm{~cm}^{-1}$ associated with the C-N of PIL-1 appeared in the spectrum of PILCs (Fig. 4a vs. Fig. 4c-e), suggesting formation of the complex was successful. The bands at about $2947 \mathrm{~cm}^{-1}$ corresponded to the asymmetric stretching vibration of the $\mathrm{N}-\mathrm{H}$ which also proved that L-proline exited in the polymer complex network (Fig. 4c-f). In addition, because of the formation of hydrogen bonds or ionic interactions between the carboxyl groups of L-proline and ionic groups of PIL, the bands at $3310 \mathrm{~cm}^{-1}$ corresponding to the asymmetric stretching vibration of the $\mathrm{OH}$ group in L-proline also shifted to a lower position. ${ }^{24}$ Meanwhile, the characteristic band at $850 \mathrm{~cm}^{-1}$ was related to the $\mathrm{P}-\mathrm{F}$ of the $\mathrm{PF}_{6}{ }^{-}$group (Fig. $4 \mathrm{~b}$ vs. Fig. $4 \mathrm{f}$ ), which proved that PIL-2 exited in PILC-4.

Elemental analysis (Table 1) of the PILCs revealed they had a stoichiometric composition in accordance with the

Table 3 Effect of solvents on reactions at $80^{\circ} \mathrm{C}$ for $21 \mathrm{~h}$

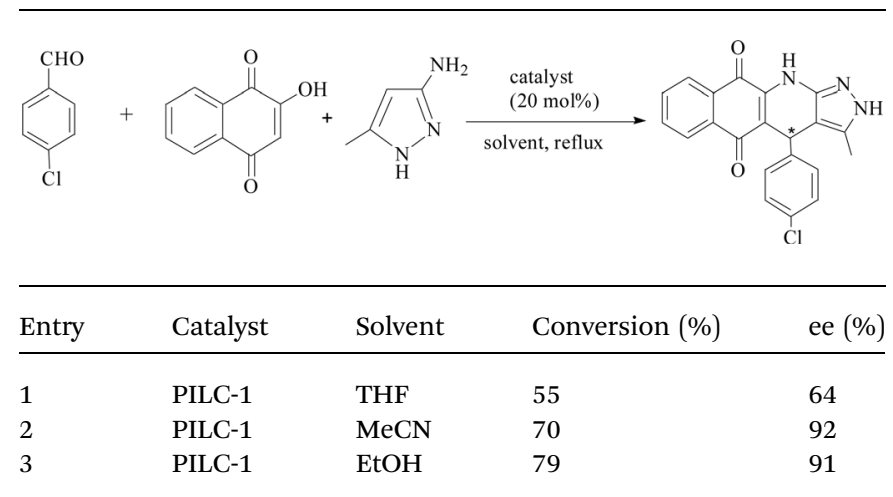


Table 4 Comparison of different catalyst systems in three component reactions between 4-chlorobenzaldehyde, 2-hydroxy-1,4-naphthoquinone, and 3-amino-5-4-methylpyrazole at $80^{\circ} \mathrm{C}$ in ethanol

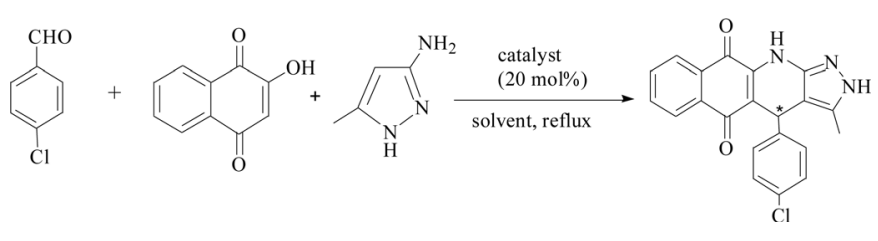

\begin{tabular}{lllll}
\hline & & $\begin{array}{l}\text { Reaction } \\
\text { time (h) }\end{array}$ & $\begin{array}{l}\text { Conversion }^{a} \\
(\%)\end{array}$ & ee $^{b}(\%)$ \\
\hline 1 & Proline & 21 & 70 & 75 \\
2 & Proline pyridinium salt & 21 & 69 & 67 \\
3 & Proline + PIL-1 & 21 & 71 & 75 \\
4 & PILC-1 & 21 & 79 & 91 \\
5 & PILC-2 & 21 & 72 & 97 \\
6 & PILC-3 & 21 & $\mathbf{9 2}$ & $\mathbf{9 8}$ \\
7 & PILC-4 & 21 & 60 & $\mathbf{9 9}$
\end{tabular}

${ }^{a}$ Determined by ${ }^{1} \mathrm{H}$ NMR spectroscopic analysis of the product. ${ }^{b}$ Determined by HPLC using a chiral column.

experimental ratios. In addition, the load of the catalyst of the heterogeneous system can be obtained by elemental analysis. XPS spectra were used to determine the surface chemical properties of the PILCs, as shown in Fig. 5. No characteristics peaks of $\mathrm{Br}$ in PILC-1 and PILC-2 are found which suggests an anion exchange on PIL-1 with the carboxylate of (L)-proline (species B, Scheme 1) but not seems to an effective solvating the (L)-proline by PIL-1 (species A, Scheme 1). ${ }^{34} \mathrm{Br}$ was in PILC-3, which can be attributed to the addition of a high ratio of PIL1. $F$ and $P$ had also appeared in PILC-4 due to the anion exchange on PIL-2 with carboxylate of (L)-proline and more PIL-2 exiting in complex network.

\section{Catalytic performance}

A typical aldol reaction between 4-nitrobenzaldehyde and acetone catalyzed by L-proline, proline pyridinium salt (synthesis in ESI $\dagger$ ) and the complex network system $(10 \mathrm{~mol} \%$ of 4 - nitrobenzaldehyde) in DMF was stirred at $25{ }^{\circ} \mathrm{C}$ for $24 \mathrm{~h}$. The reaction results are presented in Table 2 . The catalytic activity of the PILCs was significantly higher than that of L-proline and proline pyridinium salt. The conversion rates of PILC-1, PILC-2, and PILC- 3 were above $80 \%$, while the conversion rates of the small molecule catalyst (L-proline) and proline pyridinium salt were only $55 \%$ and $35 \%$. The reaction rates demonstrate that the PIL network catalysts have the excellent catalytic activity. Compared to L-proline (76\% ee value) and proline pyridinium salt (71\% ee value), the $77 \%$ ee value of PILC- 3 indicated that the optical activity of L-proline was maintained in the polymer network. The decreasing ee of the other PILCs (PILC-1, PILC-2, and PILC-4) revealed the flaws in the heterogeneous catalytic system. ${ }^{10}$ We also added an equal quantity of PIL-1 to L-proline DMF solution to study the effect of PIL on catalytic performance. However, the catalytic activity and enantioselectivity were unaltered compared with the pure L-proline. These results suggest the interactions between PIL and L-proline had no effect on catalytic performance in a free solution state. Compared to proline pyridinium salt, the improved catalytic activity and enantioselectivity of PILC-3 can be attributed to the newly confined complex network structure and the synergistic effect of the PIL. This also highlights the capacity of PIL additive to solvate $\mathrm{L}^{-}$ proline molecule avoiding proline aggregates and favoring increasing the catalytic activity. By using a large excess of PIL, the supramolecular interactions result in a homogeneous dispersion for L-proline in the polymer network, to which PIL provides an appropriate mass transfer for the catalytic process.

Furthermore, the new system was used to catalyze the relatively complicated three-component reactions of 4-chlorobenzaldehyde with 2-hydroxy-1,4-naphthoquinone and 3amino-5-4-methylpyrazole. Heterocyclic compounds are very important organic compounds widely used in the fields of medicine, ${ }^{36}$ pesticides, ${ }^{37}$ and other materials that are synthesized by MCRs. ${ }^{38,39}$ PILC-1 was first used to catalyst threecomponent reactions in an acetonitrile, ethanol, and THF system (Table 3). Interesting, catalytic activity and enantioselectivity were the highest for the PILC-1 in ethanol as it had the optimal conversion (79\%) and ee value (91\%) among the solvents (Table 3 , entry 3 ).

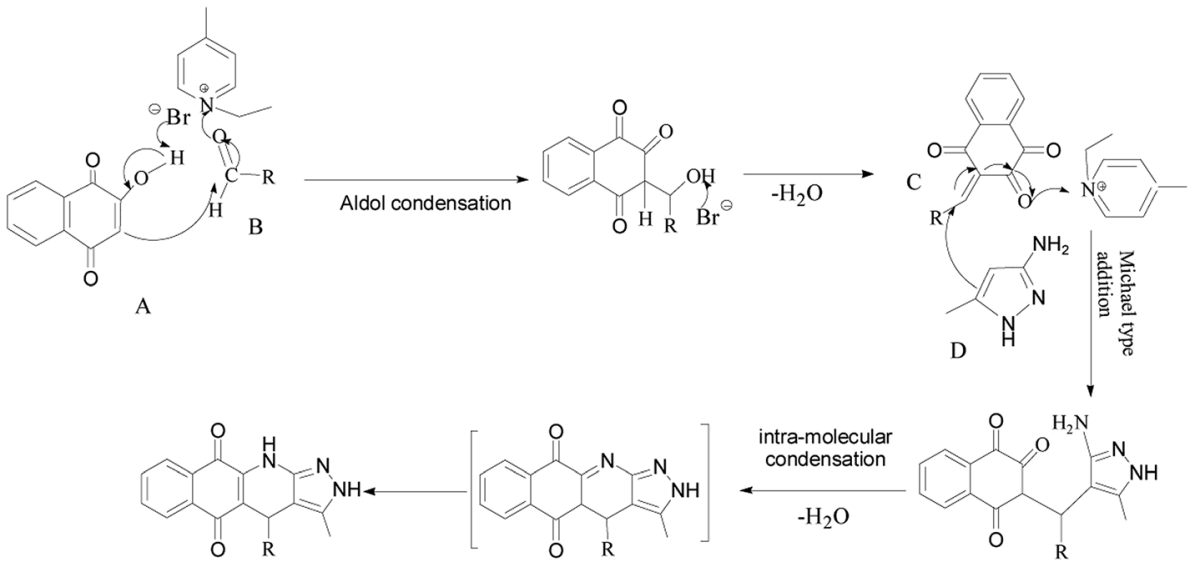

Scheme 2 Proposed mechanism for the synthesis of $2 H$-benzo[g]-pyrazolo[3,4-b]quinoline-5,10(4H,11H)-diones. 
To determine the effect of different structures on the catalytic properties of PILCs, three component reactions were carried out with different catalyst systems (Table 4). L-Proline, which had a $70 \%$ conversion and $75 \%$ ee value at $21 \mathrm{~h}$, and proline pyridinium salt (69\% conversion and $67 \%$ ee value) were used as the reference (Table 4, entry 1 and entry 2). The addition of PIL to the reaction had no obvious effect on the catalytic activity and enantioselectivity of L-proline as the conversion was $71 \%$ and the ee value was $75 \%$. PILC-1 had better catalytic activity and enantioselectivity (75\% conversion rate and $91 \%$ ee) than L-proline and proline pyridinium salt, which showed that the layer structure provided a more favorable catalytic environment and improved the catalytic properties. PILC-2 (72\% conversion rate and $97 \%$ ee) displayed higher selectivity than PILC-1. PILC-3 had the best conversion rate (92\%) and ee value (98\%), meaning that more PIL-1 has the synergic effects and improved the catalytic properties. The polymer network acted not only as a better support for L-proline, but also as a promoter to promote the catalytic activity and enantioselectivity. The catalysts combining the merits of the synergic effects of PIL and the confined effects of this polymer network provided efficient heterogeneous catalytic systems. However, PILC-4 displayed poor catalytic activity ( $60 \%$ conversion rate), but good enantioselectivity (99\% ee). The hydrophobic PIL structure did not aid in the reaction substrate entering the system and, thus, inhibited the reaction. An advantage of PILCs as catalysts is that the catalytic properties can be adjusted by changing the PIL anion. In addition, PILC-3 shows the excellent catalytic properties, a plausible mechanism for PILC-3 catalyzed one-pot MCR is outlined in Scheme $2 .^{\mathbf{4 0 4 1}}$ As a donor, the $\mathrm{Br}$ anion of the IL activated the $\mathrm{O}-\mathrm{H}$ of $\mathrm{A}$, and the cation of the IL activated the $\mathrm{C}=\mathrm{O}$ of $\mathrm{B}$ as a electronic acceptor. This synergistic effect of the PIL led to the addition of one molecule of A to one molecule B. The next step involved a Michael addition of $\mathrm{D}$ to the $\mathrm{C}=\mathrm{C}$ bond of $\mathrm{C}$, which was also activated by the anion of the ionic liquid, leading to the target compound with high efficiency. In this catalytic procedure, the cation and the anion of PIL-1 have

Table 5 The three component reactions between different substitution at $80^{\circ} \mathrm{C}$ with PILC-3 as catalyst

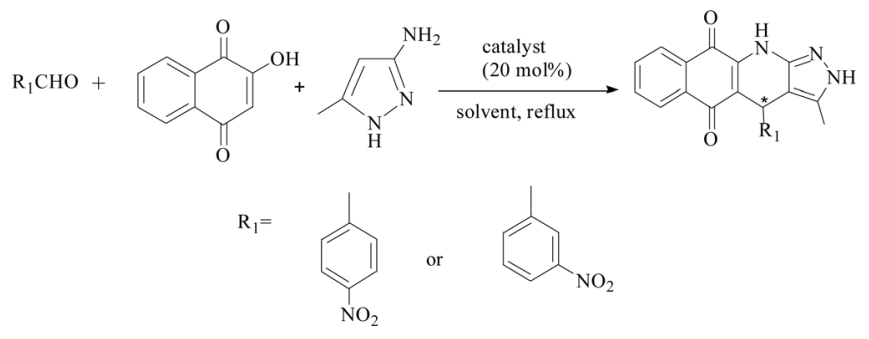

\begin{tabular}{lllll} 
& Solvent & $\begin{array}{l}\text { Reaction time } \\
(\mathrm{h})\end{array}$ & $\begin{array}{l}\text { Conversion } \\
(\%)\end{array}$ & ee (\%) \\
\hline $\mathrm{R}_{1}$ & & & 97 & 87 \\
$p$-Nitrobenzaldehyde & EtOH & 21 & 70 & 82 \\
$m$-Nitrobenzaldehyde & EtOH & 21 & 85 & 74 \\
$p$-Nitrobenzaldehyde & MeCN & 21 & 80 & 68 \\
$m$-Nitrobenzaldehyde & MeCN & 21 & &
\end{tabular}

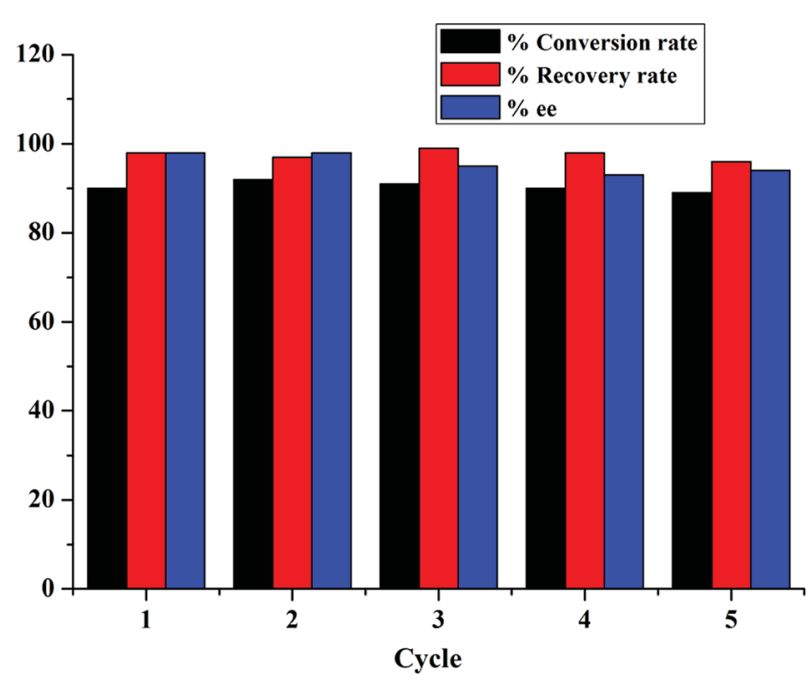

Fig. 6 Three component reactions between 3-amino-5-4-methylpyrazole, 2-hydroxy-1,4-naphthoquinone, and 4-chlorobenzaldehyde catalyzed by PILC-3 in ethanol for $21 \mathrm{~h}$ in multiple cycles.

a synergetic effect on the substrates. This is a possible reason why PILC-3 could catalyze MCRs more effectively.

The three-component reactions were also extended to other several substituted benzaldehydes, including 2-hydroxy-1,4naphthoquinone and 3-amino-5-4-methylpyrazole, and the results are presented in Table 5. PILC-3 effectively promoted reactions with a variety of aromatic aldehydes (e.g., p-nitrobenzaldehyde), where the products had better conversion (97\%) and ee value (87\%) in ethanol than in MeCN (Table 5).

Furthermore, PILC-3 was successfully used in the three component reaction as a catalyst for 5 cycles with no discernible decrease in activity and enantioselectivity (Fig. 6). The elemental analysis result also proved that L-proline had no loss during consecutive cycles (Table 1).

\section{Conclusions}

A new heterogeneous proline catalyst system was prepared based on the strong noncovalent bonds between PIL and $\mathrm{L}^{-}$ proline and the further free radical polymerization of $\mathrm{L}_{\mathrm{L}}$ proline monomer. Due to the different reaction rates of PIL and $\mathrm{L}^{-}$ proline, as well as different PIL anions, different polymer network structures were formed. The heterogeneous catalyst system could catalyze direct asymmetric aldol reactions and MCRs efficiently and had better catalytic activity and enantioselectivity than homogeneous counterparts. The catalytic system not only efficiently loaded the chiral catalyst simply using the noncovalent approach, the unique network structures and synergic effects of PIL also resulted in high catalytic performance for the heterogeneous L-proline. This catalytic system was able to be reused and recycled five times with no discernible loss in catalytic activity and enantioselectivity. This method of synthesizing polymer network-supported catalysts provides a new approach by which to prepare high performance supported catalysts for organic reactions. 


\section{Conflicts of interest}

There are no conflicts to declare.

\section{Acknowledgements}

This work was supported by the National Natural Science Foundation of China (No. 21204019), the Post-doctoral Foundation of China (No. 2012M521398), the National Natural Science Foundation of Henan Province (No. 5201039130118), the Key Scientific Research Projects in 18 Colleges and Universities (No. 5201039140142).

\section{References}

1 B. List, R. A. Lerner and C. F. Barbas, J. Am. Chem. Soc., 2000, 122, 2395-2396.

2 X. J. Li, M. Q. Chen, B. L. Yang, S. L. Zhang, X. B. Jia and Z. G. Hu, RSC Adv., 2014, 4, 43278-43285.

3 X. J. Li, C. N. Lv, X. B. Jia, M. Q. Chen, K. Wang and Z. G. Hu, ACS Appl. Mater. Interfaces, 2017, 9, 827-835.

4 L. J. Liu, T. Y. Zhou and S. G. Telfer, J. Am. Chem. Soc., 2017, 139, 13936-13943.

5 J. Liu and L. Wang, Synthesis, 2016, 49, 960-972.

6 Y. Zhang, J. Zhu, N. Yu and H. Yu, Chin. J. Chem., 2015, 33, 171-174.

7 T. Baba, J. Yamamoto, K. Hayashi, M. Sato, M. Yamanaka, T. Kawabata and T. Furata, Chem. Sci., 2016, 7, 3791-3797.

8 M. Aktas, A. Uyanik, S. Eymur and M. Yilmaz, Supramol. Chem., 2016, 28, 1-9.

9 D. Zhang, A. Martinez and J. P. Dutasta, Chem. Rev., 2017, 117, 4900-4942.

10 S. Wang, P. Liu, W. Wang, Z. Zhang and B. G. Li, Catal. Sci. Technol., 2015, 5, 3798-3805.

11 X. J. Li, B. L. Yang, X. B. Jia, M. Q. Chen and Z. G. Hu, RSC Adv., 2015, 5, 89149-89156.

12 L. Zhou, B. F. Chu, X. Y. Xu, L. Xu, N. Liu and Z. Q. Wu, ACS Macro Lett., 2017, 6, 824-829.

13 H. Wang, N. Li, Z. Yan, J. Zhang and X. Wan, RSC Adv., 2015, 5, 52410-52419.

14 M. A. Pericas, I. K. Sagamanova, S. Sayalero, S. MartínezArranz and A. C. Albeniz, Catal. Sci. Technol., 2015, 5, 754764.

15 L. N. Neumann, M. B. Baker, C. M. A. Leender, I. K. Voets, R. P. Lafleur, A. R. Palmans and E. W. Meijer, Org. Biomol. Chem., 2015, 13, 7711-7719.

16 H. Liao, C. Shen, F. Shen, P. Zhang and W. Su, Curr. Organocatal., 2015, 2, 71-76.

17 G. Guo, Y. Wu, X. Zhao, J. Wang, L. Zhang and Y. Cui, Tetrahedron: Asymmetry, 2016, 27, 740-746.
18 A. P. Gerola, E. H. Wanderlind, Y. S. Gomes, L. A. Giusti, L. García-Río, R. A. Nome, A. J. Kirby, H. D. Fiedler and F. Nome, ACS Catal., 2017, 7, 2230-2239.

19 M. Ferré, X. Cattoën and R. Pleixats, ChemistrySelect, 2016, 1, 6741-6748.

20 E. Akceylan, A. Uyanik, S. Eymur, O. Sahin and M. Yilmaz, Appl. Catal., A, 2015, 499, 205-212.

21 A. A. Elmekawy, J. B. Sweeney and D. R. Brown, Catal. Sci. Technol., 2015, 5, 690-696.

22 D. D. Chronopoulos, C. G. Kokotos, N. Karousis, G. Kokotos and N. Tagmatarchis, Nanoscale, 2015, 7, 2750-2757.

23 L. González, J. Escorihuela, B. Altava, M. I. Burguete and S. V. Luis, Eur. J. Org. Chem., 2015, 2014, 5356-5363.

24 M. Zhang, R. Ettelaie, T. Yan, S. J. Zhang, F. Q. Cheng, B. P. Binks and H. Q. Yang, J. Am. Chem. Soc., 2017, 139, 17387-17396.

25 D. E. Siyutkin, A. S. Kucherenko and S. G. Zlotin, Tetrahedron, 2009, 65, 1366-1372.

26 D. E. Siyutkin, A. S. Kucherenko and S. G. Zlotin, Tetrahedron, 2010, 66, 513-518.

27 A. Obregónzúñiga, M. Milán and E. Juaristi, Org. Lett., 2017, 19, 1108-1111.

28 R. Porcar, M. I. Burguete, P. Lozano, E. Garciaverdugo and S. V. Luis, ACS Sustainable Chem. Eng., 2016, 4, 6062-6071.

29 X. Liu, C. Chen, Y. Xiu, A. Chen, L. Guo, R. Zhang, J. Z. Chen and Z. S. Hou, Catal. Commun., 2015, 67, 90-94.

30 R. Alimohammadzadeh and M. Galehassadi, Lett. Org. Chem., 2016, 13, 496-503.

31 Z. An, W. H. Zhang, H. M. Shi and J. He, J. Catal., 2006, 241, 319-327.

32 R. Tan, C. Li, J. Luo, Y. Kong, W. Zheng and D. Yin, J. Catal., 2013, 298, 138-147.

33 W. F. Zhang, Z. Li, H. Y. Gu, Y. Li, G. L. Zhang, F. B. Zhang and X. B. Fan, Chem. Eng. Sci., 2015, 135, 187-192.

34 X. J. Li, K. Wang, N. N. Ma and X. B. Jia, Front. Chem., 2017, 5, 69.

35 S. Nießing, C. Czekelius and C. Janiak, Catal. Commun., 2017, 95, 12-15.

36 S. Karamthulla, S. Pal, T. Parvin and L. H. Choudhury, RSC Adv., 2014, 4, 15319-15324.

37 A. Khalafi-Nezhad, S. Sarikhani, E. S. Shahidzadeh and F. Panahi, Green Chem., 2012, 14, 2876-2884.

38 G. Martinez-Ariza, N. McConnell and C. Hulme, Org. Lett., 2016, 18, 1864-1867.

39 Q. H. Zhang, S. J. Zhang and Y. Q. Deng, Green Chem., 2011, 13, 2619-2637.

40 H. G. O. Alvim, D. L. J. Pinheiro, V. H. Carvalho-Silva, M. Fioramonte, F. C. Gozzo, W. A. Silva, G. W. Amarante and B. A. D. Neto, J. Org. Chem., 2018, 83, 12143-12153.

41 A. L. Zhu, R. X. Liu, C. Y. Du and L. J. Li, $R S C A d v .$, 2017, 7, 6679-6684. 